\title{
Blowup Phenomena for a Modified Dullin-Gottwald-Holm Shallow Water System
}

\author{
Panpan Zhai, Zhengguang Guo, and Weiming Wang \\ College of Mathematics and Information Science, Wenzhou University, Wenzhou 325035, China \\ Correspondence should be addressed to Weiming Wang; weimingwang2003@163.com
}

Received 6 April 2013; Accepted 11 June 2013

Academic Editor: Sergey Piskarev

Copyright (C) 2013 Panpan Zhai et al. This is an open access article distributed under the Creative Commons Attribution License, which permits unrestricted use, distribution, and reproduction in any medium, provided the original work is properly cited.

We discuss blowup phenomena for a modified two-component Dullin-Gottwald-Holm shallow water system. In this paper, some new blowup criteria of strong solutions involving the density and suitable integral form of the momentum are established.

\section{Introduction}

We consider the following two-component DGH type system:

$$
\begin{gathered}
y_{t}+2 \omega u_{x}+u y_{x}+2 y u_{x}+\gamma u_{x x x}+g \rho \bar{\rho}_{x}=0, \\
y=u-\alpha^{2} u_{x x}, \\
\rho_{t}+(\rho u)_{x}=0,
\end{gathered}
$$

where $u=u(x, t),(x, t) \in\left(\mathbb{R}, \mathbb{R}^{+}\right)$denotes the velocity field, $g$ is the downward constant acceleration of gravity in applications to shallow water waves, and $\rho=\left(1-\partial_{x}^{2}\right)(\bar{\rho}-$ $\left.\bar{\rho}_{0}\right)$, where $\bar{\rho}_{0}$ is taken to be a constant. It is obvious that if $\rho \equiv 0$, then (1) reduces to the well-known Dullin-GottwaldHolm equation [1] (DGH equation for short). There are some contributions to DGH equation concerning the wellposedness, scattering problem, blowup phenomenon, and so forth; see, for example, [2-5] and references therein. We find that (1) is expressed in terms of an averaged filtered density component $\bar{\rho}$ in analogy to the relation between momentum and velocity by setting $\rho=\left(1-\partial_{x}^{2}\right)\left(\bar{\rho}-\bar{\rho}_{0}\right)$ and the velocity component $u$. The idea is actually from the recent work [6]. Our modification breaks the structure of DGH2 system derived by following Ivanov's approach [7] by the authors in [8]. The motivation of current research is stated as follows. From geometric point of view, (1) is the model for geodesic motion on the semidirect product Lie group of diffeomorphisms acting on densities, with respect to the $H^{1}$ norm of velocity $u$ and the $H^{1}$-norm on filtered density. From a physical point of view, (1) admits wave breaking phenomena in finite time which attracts researchers' interest. We also find that the $H^{1}$-norm of $(u, \bar{\rho})$ is conserved with respect to time variable. This makes further different discussions on the singularities, unlike those for the DGH2 system or two-component Camassa-Holm system, possible. In the previous works [9-11] on the two-component CamassaHolm equation and its modified version, blowup conditions were established in view of the negativity of initial velocity slope at some point; basically, the initial integral form of momentum is never involved. That is why we consider this kind of blowup condition in this paper. Precisely, we show the solutions blowup in finite time provided that the initial density and momentum satisfy certain sign conditions. To our knowledge, less results exist yet for the formation of singularities of (1) although the approaches we applied here are standard. The methods in previous works cannot be moved to this model parallelly. For convenience, let $v=\bar{\rho}-\bar{\rho}_{0}$ and $\Lambda=\left(1-\alpha^{2} \partial_{x}^{2}\right)^{-1}$; then the operator $\Lambda$ can be expressed by its associated Green's function $G(x)=(1 / 2 \alpha) e^{-|x / \alpha|}$ with

$$
\Lambda f(x)=G * f(x)=\int_{\mathbb{R}} G(x-y) f(y) d y
$$

Using this identity, system (1) takes an equivalent form of a 
quasilinear evolution equation of hyperbolic type as follows:

$$
\begin{gathered}
u_{t}+u_{x}\left(u-\frac{\gamma}{\alpha^{2}}\right) \\
=-\partial_{x} G *\left(u^{2}+\frac{\alpha^{2}}{2} u_{x}^{2}+\left(2 \omega+\frac{\gamma}{\alpha^{2}}\right) u+\frac{g}{2} v^{2}-\frac{g}{2} v_{x}^{2}\right), \\
v_{t}+u v_{x}=-G *\left(\left(u_{x} v_{x}\right)_{x}+u_{x} v\right) .
\end{gathered}
$$

The current paper is based on some results on the Camassa-Holm equation [12-19] and its two-component generalizations [20-27]. We investigate further formation of singularities of solutions to (3) with the case of $g=1$ and $\alpha>$ 0 , just for simplicity mathematically. This paper is organized as follows. In Section 2, we recall some preliminary results on the well-posedness and blowup scenario. In Section 3, the detailed blowup conditions are presented.

\section{Preliminaries}

In this section, for completeness, we recall some elementary results and skip their proofs since they are not the main concern of this work. For convenience, in what follows, we let $\lambda=-\gamma / \alpha^{2}$ and $2 \kappa=2 \omega+\gamma / \alpha^{2}$.

We can apply Kato's theory [28] to establish the following local well-posedness theorem for (3).

Theorem 1. Assume an initial data $\left(u_{0}, v_{0}\right) \in H^{s} \times H^{s-1}, s \geq$ $5 / 2$. Then there exists a maximal $T=T\left(\left\|u_{0}, v_{0}\right\|_{H^{s} \times H^{s-1}}\right)>0$ and a unique solution

$$
(u, v) \in C\left([0, T) ; H^{s} \times H^{s-1}\right) \cap C^{1}\left([0, T) ; H^{s-1} \times H^{s-2}\right)
$$

of system (3). Moreover, the solution $(u, v)$ depends continuously on the initial value $\left(u_{0}, v_{0}\right)$, and the maximal time of existence $T>0$ is independent of $s$.

The proof of Theorem 1 is similar to the one in [11]. Moreover, using the techniques in [11], one can get the criterion for finite time wave breaking to (3) as follows.

Theorem 2. Let $\left(u_{0}, v_{0}\right) \in H^{s} \times H^{s-1}$ with $s \geq 5 / 2$, and let $T>0$ be the maximal time of existence of the solution $(u, v)$ to (3) with initial data $\left(u_{0}, v_{0}\right)$. Then the corresponding solution $(u, v)$ blowsup in finite time if and only if

$$
\lim _{t \uparrow T}\left\{\inf _{x \in \mathbb{R}} u_{x}(t, x)\right\}=-\infty
$$

Lemma 3 (see [29]). Assume that a differentiable function $y(t)$ satisfies

$$
y^{\prime}(t) \leq-C y^{2}(t)+K
$$

with constants $C, K>0$. If the initial datum $y(0)=y_{0}<$ $-\sqrt{K / C}$, then the solution to (9) goes to $-\infty$ before $t$ tends to $1 /\left(-C y_{0}+K / y_{0}\right)$.
Lemma 4 (see [19]). Suppose that $\Psi(t)$ is twice continuously differential satisfying

$$
\begin{gathered}
\Psi^{\prime \prime}(t) \geq C_{0} \Psi^{\prime}(t) \Psi(t), \quad t>0, C_{0}>0, \\
\Psi(t)>0, \quad \Psi^{\prime}(t)>0 .
\end{gathered}
$$

Then $\Psi$ blowsup in finite time. Moreover the blowup time can be estimated in terms of the initial datum as

$$
T \leq \max \left\{\frac{2}{C_{0} \Psi(0)}, \frac{\Psi(0)}{\Psi^{\prime}(0)}\right\} .
$$

We also need to introduce the standard particle trajectory method for later use. Consider now the following two initial value problems:

$$
\begin{gathered}
q_{1, t}=u\left(t, q_{1}\right)+\lambda, \quad t \in[0, T), \\
q_{1}(0, x)=x, \quad x \in \mathbb{R}, \\
q_{2, t}=u\left(t, q_{2}\right), \quad t \in[0, T), \\
q_{2}(0, x)=x, \quad x \in \mathbb{R},
\end{gathered}
$$

where $u \in C^{1}\left([0, T), H^{s-1}\right)$ is the first component of the solution $(u, v)$ to system (3) with initial data $\left(u_{0}, v_{0}\right) \in H^{s} \times$ $H^{s-1}(s \geq 5 / 2)$, and $T>0$ is the maximal time of existence. By direct computation, we have

$$
q_{i, t x}(t, x)=u_{x}\left(t, q_{i}(t, x)\right) q_{i, x}(t, x), \quad i=1,2 .
$$

Then,

$$
q_{i, x}(t, x)=\exp \left(\int_{0}^{t} u_{x}\left(\tau, q_{i}(\tau, x)\right) d \tau\right)>0, \quad t>0, x \in \mathbb{R}
$$

which means that $q_{i}(t, \cdot): \mathbb{R} \rightarrow \mathbb{R}$ is a diffeomorphism of the line for every $t \in[0, T)$. Consequently, the $L^{\infty}$-norm of any function $v(t, \cdot)$ is preserved under the family of the diffeomorphisms $q_{i}(t, \cdot)$; that is,

$$
\begin{array}{r}
\|v(t, \cdot)\|_{L^{\infty}}=\left\|v\left(t, q_{1}(t, \cdot)\right)\right\|_{L^{\infty}}=\left\|v\left(t, q_{2}(t, \cdot)\right)\right\|_{L^{\infty}}, \\
t \in[0, T) .
\end{array}
$$

Similarly,

$$
\begin{array}{r}
\inf _{x \in \mathbb{R}} v(t, x)=\inf _{x \in \mathbb{R}} v\left(t, q_{1}(t, x)\right)=\inf _{x \in \mathbb{R}} v\left(t, q_{2}(t, x)\right), \\
t \in[0, T), \\
\sup _{x \in \mathbb{R}} v(t, x)=\sup _{x \in \mathbb{R}} v\left(t, q_{1}(t, x)\right)=\sup _{x \in \mathbb{R}} v\left(t, q_{2}(t, x)\right), \\
t \in[0, T) .
\end{array}
$$

\section{Blowup Phenomenon}

In this section, we show that blowup phenomenon is the only one way that singularity arises in smooth solutions. We start this section with the following useful lemma. 
Lemma 5. Let $X_{0}=\left(u_{0}, v_{0}\right) \in H^{s} \times H^{s-1}, s \geq 2$. T is assumed to be the maximal existence time of the solution $X=(u, v)$ to system (3) corresponding to the initial data $X_{0}$. Then for all $t \in[0, T)$, one has the following conservation law:

$$
E(t)=\int_{\mathbb{R}}\left(u^{2}+\alpha^{2} u_{x}^{2}+v^{2}+v_{x}^{2}\right) d x
$$

Proof. We will prove that $E(t)$ is a conserved quantity with respect to time variable. Here we use the classical energy method. Multiplying the first equation in (3) by $u(x, t)$ and integrating by parts, we obtain

$$
\int_{\mathbb{R}} u u_{t} d x+\int_{\mathbb{R}} \alpha^{2} u_{x} u_{x t} d x=-\int_{\mathbb{R}} u v_{x}\left(v-v_{x x}\right) d x .
$$

Similarly, we have the following inequality for the second equation (3):

$$
\int_{\mathbb{R}} v v_{t}+v_{x} v_{x t} d x=\int_{\mathbb{R}} u v_{x}\left(v-v_{x x}\right) d x .
$$

This implies that

$$
\int_{\mathbb{R}}\left(u u_{t}+\alpha^{2} u_{x} u_{x x}+v v_{t}+v_{x} v_{x x}\right) d x=0
$$

Thus, we have

$$
\begin{aligned}
& \frac{d}{d t} \int_{\mathbb{R}}\left(u^{2}+\alpha^{2} u_{x}^{2}+v^{2}+v_{x}^{2}\right) d x \\
& \quad=2 \int_{\mathbb{R}}\left(u u_{t}+\alpha^{2} u_{x} u_{x x}+v v_{t}+v_{x} v_{x x}\right) d x=0 .
\end{aligned}
$$

This completes the proof.

Using this conservation law, we obtain

$$
\begin{aligned}
& \|u(\cdot, t)\|_{L^{\infty}(\mathbb{R})}^{2}+\|v(\cdot, t)\|_{L^{\infty}(\mathbb{R})}^{2} \\
& \quad \leq \frac{1}{2 \alpha}\|u(\cdot, t)\|_{H_{\alpha}^{1}(\mathbb{R})}^{2}+\frac{1}{2}\|v(\cdot, t)\|_{H^{1}(\mathbb{R})}^{2} \leq C_{1} E(0),
\end{aligned}
$$

where

$$
C_{1}=\max \left\{\frac{1}{2 \alpha}, \frac{1}{2}\right\}
$$

Theorem 6. Suppose that $X_{0}=\left(u_{0}, v_{0}\right) \in H^{s} \times H^{s-1}, s \geq$ $5 / 2, \rho_{0}\left(x_{0}\right)=y_{0}\left(x_{0}\right)+\kappa=0$, and the initial data satisfies the following conditions:

(i) $\rho_{0}(x) \geq 0$ on $\left(-\infty, x_{0}\right)$ and $\rho_{0}(x) \leq 0$ on $\left(x_{0}, \infty\right)$,

(ii) $\int_{-\infty}^{x_{0}} e^{\xi / \alpha}\left(y_{0}(\xi)+\kappa\right) d \xi>0$ and $\int_{-\infty}^{x_{0}} e^{-\xi / \alpha}\left(y_{0}(\xi)+\right.$ $\kappa) d \xi<0$,

for some point $x_{0} \in \mathbb{R}$. Then the solution to system (3) with the initial value $X_{0}$ blowsup in finite time.
Proof. Differentiating the first equation of (3) with respect to $x$, we obtain

$$
\begin{aligned}
u_{x t}+ & u_{x}^{2}+u u_{x x}+\lambda u_{x x} \\
& +\partial_{x}^{2} G *\left(u^{2}+\frac{\alpha^{2}}{2} u_{x}^{2}+2 \kappa u+\frac{1}{2} v^{2}-\frac{1}{2} v_{x}^{2}\right)=0 .
\end{aligned}
$$

Applying the relation $\partial_{x}^{2}(G * f)=\left(1 / \alpha^{2}\right)(G * f-f)$ yields

$$
\begin{aligned}
u_{x t}+ & u_{x}^{2}+u u_{x x}+\lambda u_{x x} \\
& +\frac{1}{\alpha^{2}} G *\left(u^{2}+\frac{\alpha^{2}}{2} u_{x}^{2}+2 \kappa u+\frac{1}{2} v^{2}-\frac{1}{2} v_{x}^{2}\right) \\
& -\frac{1}{\alpha^{2}}\left(u^{2}+\frac{\alpha^{2}}{2} u_{x}^{2}+2 \kappa u+\frac{1}{2} v^{2}-\frac{1}{2} v_{x}^{2}\right)=0
\end{aligned}
$$

From (23) we have

$$
\begin{aligned}
\frac{d}{d t} u_{x} & \left(q_{1}\left(x_{0}, t\right), t\right) \\
= & \left(u_{x t}+u u_{x x}+\lambda u_{x x}\right)\left(q_{1}\left(x_{0}, t\right), t\right) \\
= & -u_{x}^{2}\left(q_{1}\left(x_{0}, t\right), t\right)-\frac{1}{\alpha^{2}} G \\
& *\left(u^{2}+\frac{\alpha^{2}}{2} u_{x}^{2}+2 \kappa u+\frac{1}{2} v^{2}-\frac{1}{2} v_{x}^{2}\right)\left(q_{1}\left(x_{0}, t\right), t\right) \\
& +\frac{1}{\alpha^{2}}\left(u^{2}+\frac{\alpha^{2}}{2} u_{x}^{2}+2 \kappa u+\frac{1}{2} v^{2}-\frac{1}{2} v_{x}^{2}\right)\left(q_{1}\left(x_{0}, t\right), t\right) \\
\leq & -\frac{1}{2} u_{x}^{2}\left(q_{1}\left(x_{0}, t\right), t\right)+\frac{1}{2 \alpha^{2}}(u+\kappa)^{2}\left(q_{1}\left(x_{0}, t\right), t\right) \\
& +\frac{1}{2 \alpha^{2}}\left(v^{2}-v_{x}^{2}\right)\left(q_{1}\left(x_{0}, t\right), t\right) \\
& -\frac{1}{2 \alpha^{2}}\left(G *\left(v^{2}-v_{x}^{2}\right)\right)\left(q_{1}\left(x_{0}, t\right), t\right),
\end{aligned}
$$

where we used the fact proved in [30] that

$$
G *\left((u+\kappa)^{2}+\frac{\alpha^{2}}{2} u_{x}^{2}\right) \geq \frac{1}{2}(u+\kappa)^{2} .
$$

In order to arrive at our result, we need the following three claims.

Claim 1. $\left.y\left(q_{1}\left(x_{0}, t\right), t\right)\right)+\kappa=0$ for all $t$ in its lifespan; $q_{1}$ is defined in (9).

It is worth noting the equivalent form of the first equation in (3) in what follows:

$$
y_{t}+u y_{x}+2 y u_{x}+\lambda y_{x}+2 \kappa u_{x}+\rho v_{x}=0
$$


From the previous equation, we can get

$$
\begin{aligned}
& \frac{d}{d t}\left(\left(y\left(q_{1}(x, t), t\right)+\kappa\right) q_{1, x}^{2}(x, t)\right) \\
& \quad=\left(y_{t}+u y_{x}+2 y u_{x}+\lambda y_{x}+2 \kappa u_{x}\right)\left(q_{1}(x, t), t\right) q_{1, x}^{2}(x, t) \\
& \quad=-\rho\left(q_{1}(x, t), t\right) v_{x}\left(q_{1}(x, t), t\right) q_{1, x}^{2}(x, t)
\end{aligned}
$$

Since $q_{2}(x, \cdot)$ defined by $(10)$ is a diffeomorphism of the line for any $t \in[0, T)$, so there exists an $x_{3}(t) \in \mathbb{R}$ such that

$$
q_{2}\left(t, x_{3}(t)\right)=q_{1}\left(t, x_{0}\right), \quad t \in[0, t) .
$$

When $t=0$, we have

$$
x_{3}(0)=q_{2}\left(0, x_{3}(0)\right)=q_{1}\left(0, x_{0}\right)=x_{0} .
$$

Now we prove that $\rho\left(t, q_{1}\left(t, x_{0}\right)\right)=0$. It is easy to get

$$
\frac{d}{d t} \rho\left(t, q_{2}\left(t, x_{3}(t)\right)\right)=-\left(\rho u_{x}\right)\left(t, q_{2}\left(t, x_{3}(t)\right)\right) \text {. }
$$

Since

$$
\rho_{0}\left(x_{0}\right)=0
$$

integrating the previous equation, we can obtain

$$
\begin{aligned}
\rho\left(t, q_{2}\left(t, x_{3}(t)\right)\right) & =\rho\left(0, q_{2}\left(0, x_{3}(0)\right)\right) e^{-\int_{0}^{t} u_{x}\left(\tau, q_{2}\left(\tau, x_{3}(\tau)\right)\right) d \tau} \\
& =\rho_{0}\left(x_{0}\right) e^{-\int_{0}^{t} u_{x}\left(\tau, q_{2}\left(\tau, x_{3}(\tau)\right)\right) d \tau}=0
\end{aligned}
$$

thus we have

$$
\rho\left(t, q_{1}\left(t, x_{0}\right)\right)=\rho\left(t, q_{2}\left(t, x_{3}(t)\right)\right)=0 .
$$

So we can get

$$
\begin{aligned}
& \frac{d}{d t}\left(\left(y\left(q_{1}\left(x_{0}, t\right), t\right)+\kappa\right) q_{1, x}^{2}\left(x_{0}, t\right)\right) \\
& \quad=-\rho\left(q_{1}\left(x_{0}, t\right), t\right) v_{x}\left(q_{1}\left(x_{0}, t\right), t\right) q_{1, x}^{2}\left(x_{0}, t\right)=0
\end{aligned}
$$

then we have

$$
y\left(q_{1}\left(x_{0}, t\right), t\right)+\kappa=y_{0}\left(x_{0}\right)+\kappa=0
$$

Our claim is proved.
Claim 2. For any fixed $t, v_{x}^{2}(x, t)-v^{2}(x, t) \leq v_{x}^{2}\left(q_{1}\left(x_{0}, t\right), t\right)-$ $v^{2}\left(q_{1}\left(x_{0}, t\right), t\right)$ for all $x \in \mathbb{R}$. For any fixed $t$, if $x \leq q_{1}\left(x_{0}, t\right)$, then

$$
\begin{aligned}
v_{x}^{2}(x, t) & -v^{2}(x, t) \\
= & -\left(\int_{-\infty}^{q_{1}\left(x_{0}, t\right)} e^{\xi} \rho(\xi, t) d \xi-\int_{x}^{q_{1}\left(x_{0}, t\right)} e^{\xi} \rho(\xi, t) d \xi\right) \\
& \times\left(\int_{q_{1}\left(x_{0}, t\right)}^{\infty} e^{-\xi} \rho(\xi, t) d \xi+\int_{x}^{q_{1}\left(x_{0}, t\right)} e^{-\xi} \rho(\xi, t) d \xi\right) \\
= & v_{x}^{2}\left(q_{1}\left(x_{0}, t\right), t\right)-v^{2}\left(q_{1}\left(x_{0}, t\right), t\right) \\
& -\int_{-\infty}^{x} e^{\xi} \rho(\xi, t) d \xi \int_{x}^{q_{1}\left(x_{0}, t\right)} e^{-\xi} \rho(\xi, t) d \xi \\
& +\int_{x}^{q_{1}\left(x_{0}, t\right)} e^{\xi} \rho(\xi, t) d \xi \int_{q_{1}\left(x_{0}, t\right)}^{\infty} e^{-\xi} \rho(\xi, t) d \xi \\
\leq & v_{x}^{2}\left(q_{1}\left(x_{0}, t\right), t\right)-v^{2}\left(q_{1}\left(x_{0}, t\right), t\right),
\end{aligned}
$$

where the condition (i) is used. Similarly, for $x \geq q_{1}\left(x_{0}, t\right)$, we also have

$$
v_{x}^{2}(x, t)-v^{2}(x, t) \leq v_{x}^{2}\left(q_{1}\left(x_{0}, t\right), t\right)-v^{2}\left(q_{1}\left(x_{0}, t\right), t\right) .
$$

So Claim 2 is proved. Consequently, we can obtain

$$
\begin{aligned}
(G * & \left.\left(v^{2}-v_{x}^{2}\right)\right)\left(q_{1}\left(x_{0}, t\right), t\right) \\
& =\frac{1}{2 \alpha} \int_{\mathbb{R}} e^{-\left|q_{1}\left(x_{0}, t\right)-\xi\right| / \alpha}\left(v^{2}-v_{x}^{2}\right)(\xi, t) d \xi \\
& \geq \frac{1}{2 \alpha} \int_{\mathbb{R}} e^{-\left|q_{1}\left(x_{0}, t\right)-\xi\right| / \alpha}\left(v^{2}-v_{x}^{2}\right)\left(q_{1}\left(x_{0}, t\right), t\right) d \xi \\
& =\left(v^{2}-v_{x}^{2}\right)\left(q_{1}\left(x_{0}, t\right), t\right) .
\end{aligned}
$$

Thus, one can get

$$
\begin{aligned}
\frac{d}{d t} u_{x}\left(q_{1}\left(x_{0}, t\right), t\right) \leq & -\frac{1}{2} u_{x}^{2}\left(q_{1}\left(x_{0}, t\right), t\right) \\
& +\frac{1}{2 \alpha^{2}}(u+\kappa)^{2}\left(q_{1}\left(x_{0}, t\right), t\right)
\end{aligned}
$$

Claim 3. $(u+\kappa)^{2}\left(q_{1}\left(x_{0}, t\right), t\right)<\alpha^{2} u_{x}^{2}\left(q_{1}\left(x_{0}, t\right), t\right)$ for all $t \geq 0$. Furthermore, $u_{x}\left(q_{1}\left(x_{0}, t\right), t\right)<0$ is strictly decreasing. 
Abstract and Applied Analysis

5

Suppose that there exists a $t_{0}$ such that $(u+\kappa)^{2}\left(q_{1}\left(x_{0}\right.\right.$, $t), t)<\alpha^{2} u_{x}^{2}\left(q_{1}\left(x_{0}, t\right), t\right)$ on $\left[0, t_{0}\right)$ and $(u+\kappa)^{2}\left(q_{1}\left(x_{0}, t_{0}\right), t_{0}\right)=$ $\alpha^{2} u_{x}^{2}\left(q_{1}\left(x_{0}, t_{0}\right), t_{0}\right)$. From the expression of $u(x, t)$ in terms of $y(x, t)$, we can rewrite $u(x, t)+\kappa$ and $u_{x}(x, t)$ as follows:

$$
\begin{aligned}
u(x, t)+\kappa= & \frac{1}{2 \alpha} e^{-x / \alpha} \int_{-\infty}^{x} e^{\xi / \alpha}(y(\xi, t)+\kappa) d \xi \\
& +\frac{1}{2 \alpha} e^{x / \alpha} \int_{x}^{\infty} e^{-\xi / \alpha}(y(\xi, t)+\kappa) d \xi \\
u_{x}(x, t)= & -\frac{1}{2 \alpha^{2}} e^{-x / \alpha} \int_{-\infty}^{x} e^{\xi / \alpha}(y(\xi, t)+\kappa) d \xi \\
& +\frac{1}{2 \alpha^{2}} e^{x / \alpha} \int_{x}^{\infty} e^{-\xi / \alpha}(y(\xi, t)+\kappa) d \xi .
\end{aligned}
$$

Letting

$$
\begin{gathered}
I(t)=e^{-q_{1}\left(x_{0}, t\right) / \alpha} \int_{-\infty}^{q_{1}\left(x_{0}, t\right)} e^{\xi / \alpha}(y(\xi, t)+\kappa) d \xi, \\
I I(t)=e^{q_{1}\left(x_{0}, t\right) / \alpha} \int_{q_{1}\left(x_{0}, t\right)}^{\infty} e^{-\xi / \alpha}(y(\xi, t)+\kappa) d \xi,
\end{gathered}
$$

then

$$
\begin{aligned}
\frac{d I(t)}{d t}= & -\frac{1}{\alpha}\left(u\left(q_{1}\left(x_{0}, t\right), t\right)+\lambda\right) e^{-q_{1}\left(x_{0}, t\right) / \alpha} \\
& \times \int_{-\infty}^{q_{1}\left(x_{0}, t\right)} e^{\xi / \alpha}(y(\xi, t)+\kappa) d \xi \\
& +e^{-q_{1}\left(x_{0}, t\right) / \alpha} \int_{-\infty}^{q_{1}\left(x_{0}, t\right)} e^{\xi / \alpha} y_{t}(\xi, t) d \xi .
\end{aligned}
$$

Integrating by parts, the first term of (42) yields

$$
\begin{aligned}
-\frac{1}{\alpha}( & \left.u\left(q_{1}\left(x_{0}, t\right), t\right)+\lambda\right) e^{-q_{1}\left(x_{0}, t\right) / \alpha} \\
& \times \int_{-\infty}^{q_{1}\left(x_{0}, t\right)} e^{\xi / \alpha}(y(\xi, t)+\kappa) d \xi \\
= & \left(\alpha u u_{x}-u^{2}-\kappa u\right)\left(q_{1}\left(x_{0}, t\right), t\right) \\
& -\frac{1}{\alpha} e^{-q_{1}\left(x_{0}, t\right) / \alpha} \int_{-\infty}^{q_{1}\left(x_{0}, t\right)} e^{\xi / \alpha} \lambda(y(\xi, t)+\kappa) d \xi .
\end{aligned}
$$

For the second term of (42), we have the following equation in the view of Claim 1:

$$
\begin{aligned}
& e^{-q_{1}\left(x_{0}, t\right) / \alpha} \int_{-\infty}^{q_{1}\left(x_{0}, t\right)} e^{\xi / \alpha} y_{t}(\xi, t) d \xi \\
&=-e^{-q_{1}\left(x_{0}, t\right) / \alpha} \int_{-\infty}^{q_{1}\left(x_{0}, t\right)} e^{\xi / \alpha}\left(((y+\kappa) u)_{x}+\frac{1}{2}\left(u^{2}-\alpha^{2} u_{x}^{2}\right)_{x}\right. \\
&\left.+\lambda(y+\kappa)_{x}+\kappa u_{x}+\rho v_{x}\right) d \xi
\end{aligned}
$$

$$
\begin{aligned}
& =\left(\frac{\alpha^{2}}{2} u_{x}^{2}-\frac{1}{2} u^{2}-\kappa u\right)\left(q_{1}\left(x_{0}, t\right), t\right) \\
& -e^{-q_{1}\left(x_{0}, t\right) / \alpha} \int_{-\infty}^{q_{1}\left(x_{0}, t\right)} e^{\xi / \alpha} \rho v_{x} d \xi \\
& +\frac{1}{\alpha} e^{-q_{1}\left(x_{0}, t\right) / \alpha} \int_{-\infty}^{q_{1}\left(x_{0}, t\right)} e^{\xi / \alpha}\left((y+\kappa) u+\frac{1}{2}\left(u^{2}-\alpha^{2} u_{x}^{2}\right)\right. \\
& =\left(\frac{\alpha^{2}}{2} u_{x}^{2}-\frac{1}{2} u^{2}-\kappa u+\frac{1}{2}\left(v_{x}^{2}-v^{2}\right)\right)\left(q_{1}\left(x_{0}, t\right), t\right) \\
& +\frac{1}{2 \alpha} e^{-q_{1}\left(x_{0}, t\right) / \alpha} \int_{-\infty}^{q_{1}\left(x_{0}, t\right)} e^{\xi / \alpha}\left(v^{2}-v_{x}^{2}\right) d \xi \\
& +\frac{1}{\alpha} e^{-q_{1}\left(x_{0}, t\right) / \alpha} \int_{-\infty}^{q_{1}\left(x_{0}, t\right)} e^{\xi / \alpha}\left(\frac{3}{2} u^{2}-\frac{\alpha^{2}}{2} u_{x}^{2}-\alpha^{2} u u_{x x}\right. \\
& +\frac{1}{\alpha} e^{-q_{1}\left(x_{0}, t\right) / \alpha} \int_{-\infty}^{q_{1}\left(x_{0}, t\right)} e^{\xi / \alpha}\left(u^{2}+\frac{\alpha^{2}}{2} u_{x}^{2}\right. \\
& +\frac{1}{2 \alpha} e^{-q_{1}\left(x_{0}, t\right) / \alpha} \int_{-\infty}^{q_{1}\left(x_{0}, t\right)} e^{\xi / \alpha}\left(v^{2}-v_{x}^{2}\right) d \xi \\
& =\left(\frac{\alpha^{2}}{2} u_{x}^{2}-\alpha u u_{x}-\kappa u+\kappa(y)+\kappa u\right) d \xi \\
& \left.\left.+v_{x}^{2}-v^{2}\right)\right)\left(q_{1}\left(x_{0}, t\right), t\right)
\end{aligned}
$$

Here we have used

$$
\begin{aligned}
-\alpha e^{-q_{1}\left(x_{0}, t\right) / \alpha} \int_{-\infty}^{q_{1}\left(x_{0}, t\right)} e^{\xi / \alpha}\left(u u_{x x}\right)(\xi, t) d \xi \\
=-\alpha\left(u u_{x}\right)\left(q_{1}\left(x_{0}, t\right), t\right)+\frac{1}{2} u^{2}\left(q_{1}\left(x_{0}, t\right), t\right) \\
+\alpha e^{-q_{1}\left(x_{0}, t\right) / \alpha} \int_{-\infty}^{q_{1}\left(x_{0}, t\right)} e^{\xi / \alpha}\left(u_{x}^{2}-\frac{1}{2 \alpha^{2}} u^{2}\right)(\xi, t) d \xi .
\end{aligned}
$$

Combining the previous equations together, and with the help of (38), (42) reads as

$$
\begin{aligned}
\frac{d I(t)}{d t}= & \left(\frac{\alpha^{2}}{2} u_{x}^{2}-u^{2}-2 \kappa u\right)\left(q_{1}\left(x_{0}, t\right), t\right) \\
& +\frac{1}{\alpha} e^{-q_{1}\left(x_{0}, t\right) / \alpha} \int_{-\infty}^{q_{1}\left(x_{0}, t\right)} e^{\xi / \alpha}\left(u^{2}+\frac{\alpha^{2}}{2} u_{x}^{2}+2 \kappa u\right) d \xi \\
& +\frac{1}{2}\left(v_{x}^{2}-v^{2}\right)\left(q_{1}\left(x_{0}, t\right), t\right)+\frac{1}{2 \alpha} e^{-q_{1}\left(x_{0}, t\right) / \alpha} \\
& \times \int_{-\infty}^{q_{1}\left(x_{0}, t\right)} e^{\xi / \alpha}\left(v^{2}-v_{x}^{2}\right) d \xi
\end{aligned}
$$




$$
\begin{aligned}
= & \left(\frac{\alpha^{2}}{2} u_{x}^{2}-(u+\kappa)^{2}\right)\left(q_{1}\left(x_{0}, t\right), t\right)+\frac{1}{\alpha} e^{-q_{1}\left(x_{0}, t\right) / \alpha} \\
& \times \int_{-\infty}^{q_{1}\left(x_{0}, t\right)} e^{\xi / \alpha}\left(\frac{\alpha^{2}}{2} u_{x}^{2}+(u+\kappa)^{2}\right) d \xi \\
& +\frac{1}{2}\left(v_{x}^{2}-v^{2}\right)\left(q_{1}\left(x_{0}, t\right), t\right)+\frac{1}{2 \alpha} e^{-q_{1}\left(x_{0}, t\right) / \alpha} \\
& \times \int_{-\infty}^{q_{1}\left(x_{0}, t\right)} e^{\xi / \alpha}\left(v^{2}-v_{x}^{2}\right) d \xi \\
\geq & \frac{1}{2}\left(\alpha^{2} u_{x}^{2}-(u+\kappa)^{2}\right)\left(q_{1}\left(x_{0}, t\right), t\right)>0, \quad \text { on }\left[0, t_{0}\right),
\end{aligned}
$$

where Claim 2 and the inequality [30]

$$
\int_{-\infty}^{x} e^{\xi / \alpha}\left((u+\kappa)^{2}+\frac{\alpha^{2}}{2} u_{\xi}^{2}\right)(\xi, t) d \xi \geq \frac{\alpha}{2} e^{x / \alpha}(u+\kappa)^{2}
$$

have been used. From the continuity property, we have

$$
\begin{array}{r}
e^{-q_{1}\left(x_{0}, t_{0}\right) / \alpha} \int_{-\infty}^{q_{1}\left(x_{0}, t_{0}\right)} e^{\xi / \alpha}\left(y\left(\xi, t_{0}\right)+\kappa\right) d \xi \\
>e^{-x_{0} / \alpha} \int_{-\infty}^{x_{0}} e^{\xi / \alpha}\left(y_{0}(\xi)+\kappa\right) d \xi>0 .
\end{array}
$$

Similarly,

$\frac{d I I(t)}{d t} \leq \frac{1}{2}\left((u+\kappa)^{2}-\alpha^{2} u_{x}^{2}\right)\left(q_{1}\left(x_{0}, t\right), t\right)<0, \quad$ on $\left[0, t_{0}\right)$.

Thus, by continuity property,

$$
\begin{array}{r}
e^{q_{1}\left(x_{0}, t_{0}\right) / \alpha} \int_{-\infty}^{q_{1}\left(x_{0}, t_{0}\right)} e^{-\xi / \alpha}\left(y\left(\xi, t_{0}\right)+\kappa\right) d \xi \\
<e^{x_{0} / \alpha} \int_{-\infty}^{x_{0}} e^{-\xi / \alpha}\left(y_{0}(\xi)+\kappa\right) d \xi<0 .
\end{array}
$$

Summarizing (48) and (50), we obtain

$$
\begin{aligned}
& \alpha^{2} u_{x}^{2}\left(q_{1}\left(x_{0}, t_{0}\right), t_{0}\right)-(u+\kappa)^{2}\left(q_{1}\left(x_{0}, t_{0}\right), t_{0}\right) \\
& =-\frac{1}{\alpha^{2}} \int_{-\infty}^{q_{1}\left(x_{0}, t_{0}\right)} e^{\xi / \alpha}\left(y\left(\xi, t_{0}\right)+\kappa\right) d \xi \\
& \quad \times \int_{q_{1}\left(x_{0}, t_{0}\right)}^{\infty} e^{-\xi / \alpha}\left(y\left(\xi, t_{0}\right)+\kappa\right) d \xi \\
& >-\frac{1}{\alpha^{2}} \int_{-\infty}^{x_{0}} e^{\xi / \alpha}\left(y_{0}(\xi)+\kappa\right) d \xi \int_{x_{0}}^{\infty} e^{-\xi / \alpha}\left(y_{0}(\xi)+\kappa\right) d \xi \\
& =\alpha^{2} u_{0 x}^{2}\left(x_{0}\right)-\left(u_{0}+\kappa\right)^{2}\left(x_{0}\right)>0 .
\end{aligned}
$$

That is a contradiction. On the other hand, from the expression of $u_{x}(x, t)$ in terms of $y(x, t)$, we can easily get that $u_{x}\left(q_{1}\left(x_{0}, t\right), t\right)<0$. So we complete the proof of Claim 3.
Furthermore, due to (46) and (49), we can obtain

$$
\begin{aligned}
\frac{d}{d t}\left(\alpha^{2} u_{x}^{2}-(u+\kappa)^{2}\right)\left(q_{1}\left(x_{0}, t\right), t\right) & \\
= & -\frac{1}{\alpha^{2}} \frac{d}{d t}\left(\int_{-\infty}^{q_{1}\left(x_{0}, t\right)} e^{\xi / \alpha}(y(\xi, t)+\kappa) d \xi\right. \\
& \left.\times \int_{q_{1}\left(x_{0}, t\right)}^{\infty} e^{-\xi / \alpha}(y(\xi, t)+\kappa) d \xi\right) \\
\geq & -\frac{1}{2 \alpha^{2}}\left(\alpha^{2} u_{x}^{2}-(u+\kappa)^{2}\right)\left(q_{1}\left(x_{0}, t\right), t\right) e^{q_{1}\left(x_{0}, t\right) / \alpha} \\
& \times \int_{-\infty}^{x} e^{-\xi / \alpha}(y(\xi, t)+\kappa) d \xi \\
& +\frac{1}{2 \alpha^{2}}\left(\alpha^{2} u_{x}^{2}-(u+\kappa)^{2}\right)\left(q_{1}\left(x_{0}, t\right), t\right) e^{-q_{1}\left(x_{0}, t\right) / \alpha} \\
& \times \int_{x}^{\infty} e^{\xi / \alpha}(y(\xi, t)+\kappa) d \xi \\
= & -u_{x}\left(q_{1}\left(x_{0}, t\right), t\right)\left(\alpha^{2} u_{x}^{2}-(u+\kappa)^{2}\right)\left(q_{1}\left(x_{0}, t\right), t\right) .
\end{aligned}
$$

Integrating (39) and then substituting it into the previous inequality, we have

$$
\begin{aligned}
& \frac{d}{d t}\left(\alpha^{2} u_{x}^{2}-(u+\kappa)^{2}\right)\left(q_{1}\left(x_{0}, t\right), t\right) \\
& \geq \frac{1}{2 \alpha^{2}}\left(\alpha^{2} u_{x}^{2}-(u+\kappa)^{2}\right)\left(q_{1}\left(x_{0}, t\right), t\right) \\
& \quad \times\left(\int_{0}^{t}\left(\alpha^{2} u_{x}^{2}-(u+\kappa)^{2}\right)\left(q_{1}\left(x_{0}, \tau\right), \tau\right) d \tau\right. \\
& \left.\quad-2 \alpha^{2} u_{0 x}\left(x_{0}\right)\right) .
\end{aligned}
$$

Let $\Psi(t)=\int_{0}^{t}\left(\alpha^{2} u_{x}^{2}-(u+\kappa)^{2}\right)\left(q_{1}\left(x_{0}, \tau\right), \tau\right) d \tau-2 \alpha^{2} u_{0 x}\left(x_{0}\right)$; then we can complete the proof with the help of Lemma 4.

Remark 7. We note that if the condition (i) is replaced by the following one:

$$
\left(i^{\prime}\right) \rho_{0}(x) \leq 0 \text { on }\left(-\infty, x_{0}\right) \text { and } \rho_{0}(x) \geq 0 \text { on }\left(x_{0}, \infty\right),
$$

then Claim 2 also holds; that is, the theorem always holds with anyone of $(\mathrm{i})$ and $\left(\mathrm{i}^{\prime}\right)$.

As a corollary of Theorem 6, we have the following.

Theorem 8. Suppose that $X_{0}=\left(u_{0}, v_{0}\right) \in H^{s} \times H^{s-1}, s \geq 5 / 2$, and the initial data satisfies the following conditions:

(i) $\rho_{0}(x) \geq 0$ on $\left(-\infty, x_{0}\right)$ and $\rho_{0}(x) \leq 0$ on $\left(x_{0}\right.$, $\infty)\left(\operatorname{or} \rho_{0}(x) \leq 0\right.$ on $\left(-\infty, x_{0}\right)$ and $\rho_{0}(x) \geq$ 0 on $\left.\left(x_{0}, \infty\right)\right)$,

(ii) $u_{0}^{\prime}\left(x_{0}\right) \leq-\left(\sqrt{C_{1} E_{0}}+|\kappa|\right) / \alpha$, 
for some point $x_{0} \in \mathbb{R}$. Then the solution to system (3) with the initial value $X_{0}$ blowsup in finite time.

Proof. As shown in Theorem 6, condition (i) guarantees that $v_{x}^{2}(x, t)+v^{2}(x, t) \leq\left(v_{x}^{2}-v^{2}\right)\left(q_{1}\left(x_{0}, t\right), t\right)$ for all $x \in \mathbb{R}$. Then,

$$
\begin{aligned}
\frac{d}{d t} u_{x}\left(q_{1}\left(x_{0}, t\right), t\right) \leq & -\frac{1}{2} u_{x}^{2}\left(q_{1}\left(x_{0}, t\right), t\right) \\
& +\frac{1}{2 \alpha^{2}}(u+\kappa)^{2}\left(q_{1}\left(x_{0}, t\right), t\right) \\
\leq & -\frac{1}{2} u_{x}^{2}\left(q_{1}\left(x_{0}, t\right), t\right) \\
& +\frac{1}{2 \alpha^{2}}\left(\sqrt{C_{1} E_{0}}+|\kappa|\right)^{2} \\
:= & -\frac{1}{2} u_{x}^{2}\left(q_{1}\left(x_{0}, t\right), t\right)+K^{2}
\end{aligned}
$$

where $K>0$ is a constant. By setting $\varphi(t)=u_{x}\left(q_{1}\left(x_{0}, t\right), t\right)$, we obtain

$$
\frac{d \varphi}{d t}=-\frac{1}{2} \varphi^{2}+K^{2}
$$

Applying Lemma 3, we have

$$
\lim _{t \uparrow T} \varphi(t)=-\infty \quad \text { with } T=\frac{1}{-(1 / 2) \varphi_{0}-\left(K^{2} / \varphi_{0}\right)},
$$

when

$$
\varphi_{0}<-\sqrt{2} K=-\frac{\sqrt{C_{1} E_{0}}+|\kappa|}{\alpha} .
$$

This completes the proof.

\section{Acknowledgments}

This work was partially supported by Natural Science Foundation of China under Grant no. 11226172 and Zhejiang Provincial Natural Science Foundation of China under Grant nos. LQ12A01009 and LY12A01014.

\section{References}

[1] H. R. Dullin, G. A. Gottwald, and D. D. Holm, "An integrable shallow water equation with linear and nonlinear dispersion," Physical Review Letters, vol. 87, no. 19, Article ID 194501, pp. 4501-4504, 2001.

[2] Y. Liu, "Global existence and blow-up solutions for a nonlinear shallow water equation," Mathematische Annalen, vol. 335, no. 3, pp. 717-735, 2006.

[3] L. Tian, G. Gui, and Y. Liu, "On the well-posedness problem and the scattering problem for the Dullin-Gottwald-Holm equation," Communications in Mathematical Physics, vol. 257, no. 3, pp. 667-701, 2005.

[4] Y. Zhou, "Blow-up of solutions to the DGH equation," Journal of Functional Analysis, vol. 250, no. 1, pp. 227-248, 2007.

[5] Y. Zhou and Z. Guo, "Blow up and propagation speed of solutions to the DGH equation," Discrete and Continuous Dynamical Systems B, vol. 12, no. 3, pp. 657-670, 2009.
[6] D. D. Holm, L. Ó Náraigh, and C. Tronci, "Singular solutions of a modified two-component Camassa-Holm equation," Physical Review E, vol. 79, no. 1, Article ID 016601, 13 pages, 2009.

[7] A. Constantin and R. I. Ivanov, "On an integrable twocomponent Camassa-Holm shallow water system," Physics Letters $A$, vol. 372, no. 48, pp. 7129-7132, 2008.

[8] F. Guo, H. Gao, and Y. Liu, "On the wave-breaking phenomena for the two-component Dullin-Gottwald-Holm system," Journal of the London Mathematical Society, vol. 86, no. 3, pp. 810834, 2012.

[9] R. M. Chen and Y. Liu, "Wave breaking and global existence for a generalized two-component Camassa-Holm system," International Mathematics Research Notices, no. 6, pp. 1381-1416, 2011.

[10] C. Guan and Z. Yin, "Global existence and blow-up phenomena for an integrable two-component Camassa-Holm shallow water system," Journal of Differential Equations, vol. 248, no. 8, pp. 2003-2014, 2010.

[11] C. Guan, K. H. Karlsen, and Z. Yin, "Well-posedness and blow-up phenomena for a modified two-component CamassaHolm equation," in Nonlinear Partial Differential Equations and Hyperbolic Wave Phenomena, vol. 526 of Contemporary Mathematics, pp. 199-220, American Mathematical Society, Providence, RI, USA, 2010.

[12] R. Camassa and D. D. Holm, "An integrable shallow water equation with peaked solitons," Physical Review Letters, vol. 71, no. 11, pp. 1661-1664, 1993.

[13] A. Constantin and J. Escher, "Wave breaking for nonlinear nonlocal shallow water equations," Acta Mathematica, vol. 181, no. 2, pp. 229-243, 1998.

[14] A. Constantin and J. Escher, "Well-posedness, global existence, and blowup phenomena for a periodic quasi-linear hyperbolic equation," Communications on Pure and Applied Mathematics, vol. 51, no. 5, pp. 475-504, 1998.

[15] A. A. Himonas, G. Misiołek, G. Ponce, and Y. Zhou, "Persistence properties and unique continuation of solutions of the Camassa-Holm equation," Communications in Mathematical Physics, vol. 271, no. 2, pp. 511-522, 2007.

[16] Z. Jiang and S. Hakkaev, "Wave breaking and propagation speed for a class of one-dimensional shallow water equations," Abstract and Applied Analysis, vol. 2011, Article ID 647368, 15 pages, 2011.

[17] H. P. McKean, "Breakdown of a shallow water equation," The Asian Journal of Mathematics, vol. 2, no. 4, pp. 867-874, 1998.

[18] Y. Zhou, "Wave breaking for a periodic shallow water equation," Journal of Mathematical Analysis and Applications, vol. 290, no. 2, pp. 591-604, 2004.

[19] Y. Zhou, "On solutions to the Holm-Staley $b$-family of equations," Nonlinearity, vol. 23, no. 2, pp. 369-381, 2010.

[20] J. Escher, O. Lechtenfeld, and Z. Yin, "Well-posedness and blowup phenomena for the 2-component Camassa-Holm equation," Discrete and Continuous Dynamical Systems A, vol. 19, no. 3, pp. 493-513, 2007.

[21] Z. Guo, "Blow-up and global solutions to a new integrable model with two components," Journal of Mathematical Analysis and Applications, vol. 372, no. 1, pp. 316-327, 2010.

[22] Z. Guo and L. Ni, "Persistence properties and unique continuation of solutions to a two-component Camassa-Holm equation," Mathematical Physics, Analysis and Geometry, vol. 14, no. 2, pp. 101-114, 2011.

[23] Z. Guo and Y. Zhou, "On solutions to a two-component generalized Camassa-Holm equation," Studies in Applied Mathematics, vol. 124, no. 3, pp. 307-322, 2010. 
[24] Z. Guo, M. Zhu, and L. Ni, "Blow-up criteria of solutions to a modified two-component Camassa-Holm system," Nonlinear Analysis. Real World Applications, vol. 12, no. 6, pp. 3531-3540, 2011.

[25] P. Zhang and Y. Liu, "Stability of solitary waves and wavebreaking phenomena for the two-component Camassa-Holm system," International Mathematics Research Notices, no. 11, pp. 1981-2021, 2010.

[26] M. Zhu, "Blow-up, global existence and persistence properties for the coupled Camassa-Holm equations," Mathematical Physics, Analysis and Geometry, vol. 14, no. 3, pp. 197-209, 2011.

[27] M. Zhu and J. Xu, "On the wave-breaking phenomena for the periodic two-component Dullin-Gottwald-Holm system," Journal of Mathematical Analysis and Applications, vol. 391, no. 2, pp. 415-428, 2012.

[28] T. Kato, "On the Cauchy problem for the (generalized) Korteweg-de Vries equation," in Studies in Applied Mathematics, vol. 8 of Advances in Mathematics, Supplementary Studies, pp. 93-128, Academic Press, New York, NY, USA, 1983.

[29] Y. Zhou, "Blow-up of solutions to a nonlinear dispersive rod equation," Calculus of Variations and Partial Differential Equations, vol. 25, no. 1, pp. 63-77, 2006.

[30] M. Zhu, L. Jin, and Z. Jiang, "A new blow-up criterion for the DGH equation," Abstract and Applied Analysis, vol. 2012, Article ID 515948, 10 pages, 2012. 


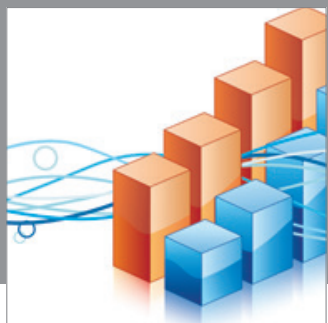

Advances in

Operations Research

mansans

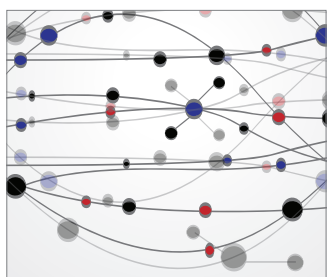

The Scientific World Journal
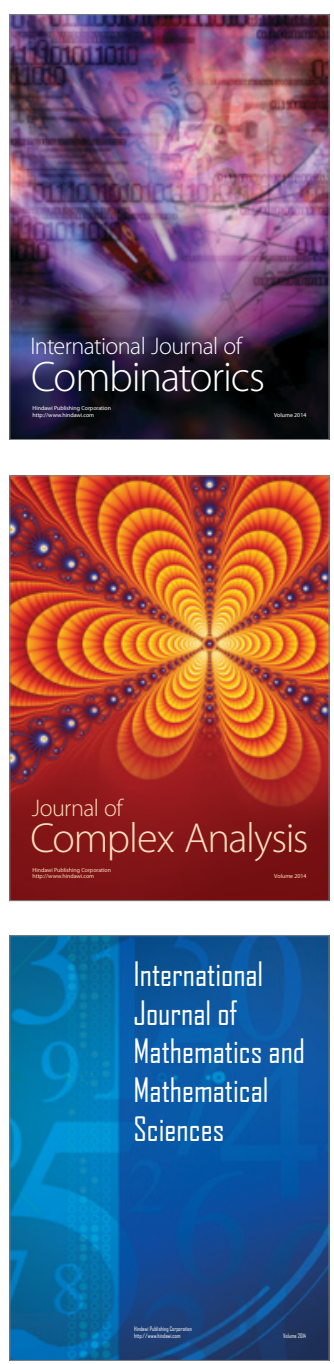
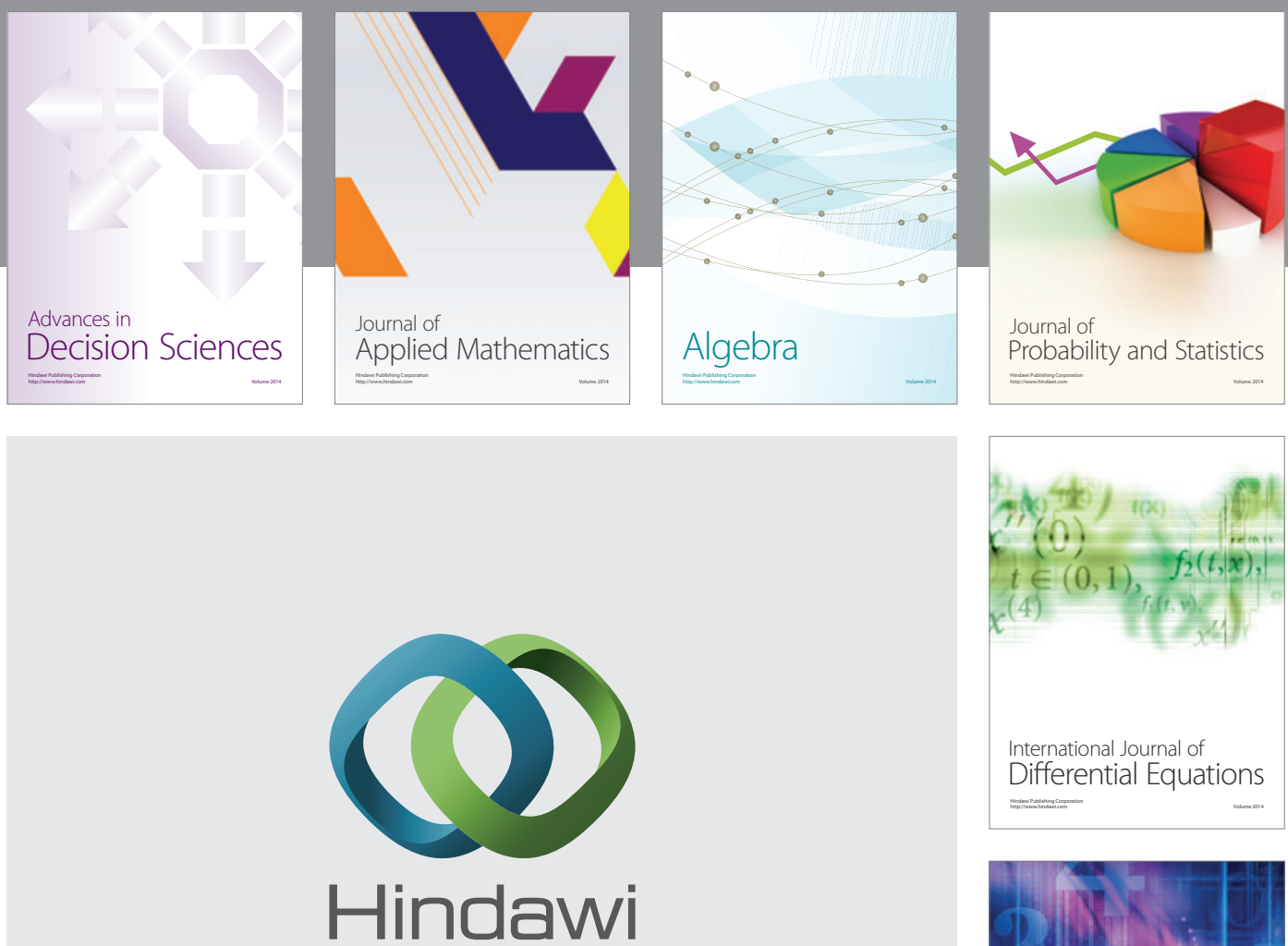

Submit your manuscripts at http://www.hindawi.com
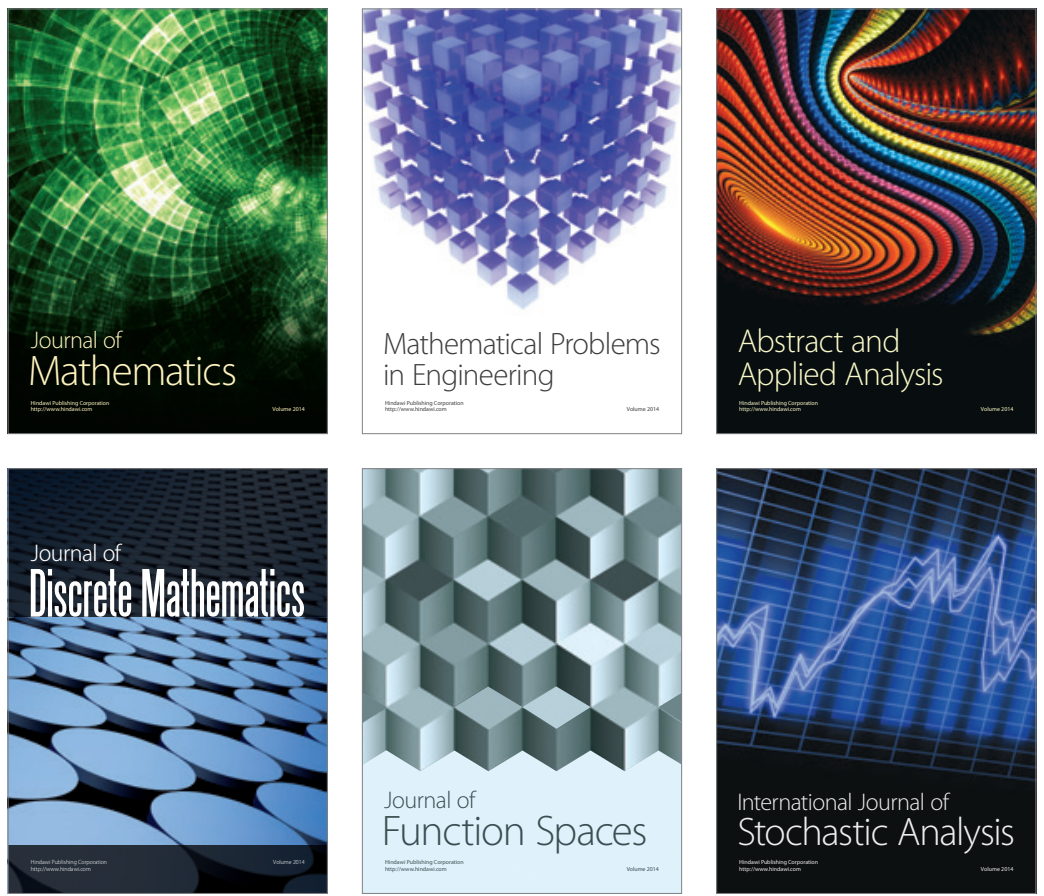

Journal of

Function Spaces

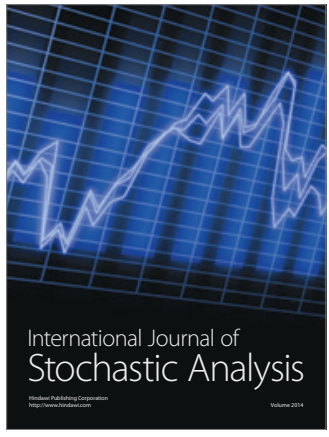

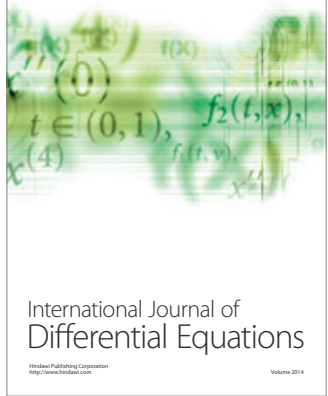
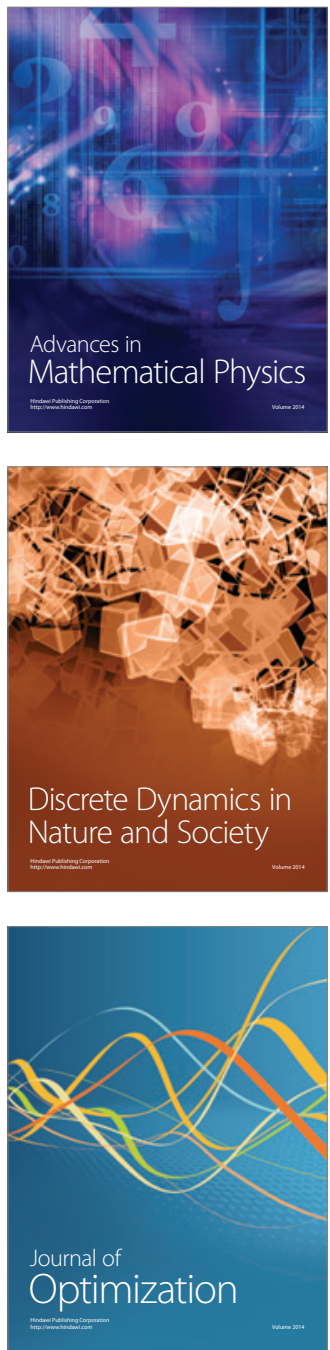